Ultrasound in Med. \& Biol., Vol. 23, No. 1, pp. 77-85, 1997 Copyright $(\mathbb{C} 1997$ World Federation for Ultrasound in Medicine \& Biology Printed in the USA. All rights reserved $0301-5629 / 97 \$ 17.00+.00$

PII S0301-5629(96)00174-3

\title{
ULTRASONIC TISSUE CHARACTERIZATION OF INFARCTED MYOCARDIUM BY SCANNING ACOUSTIC MICROSCOPY
}

\author{
Yoshifumi Saijo, * Motonao Tanaka, * Hiroaki Okawai, * Hidehiko Sasaki, * \\ SHIN-ICHI NITTA* and FLOYD DUNN ${ }^{\dagger}$ \\ *Department of Medical Engineering and Cardiology, Institute of Development, Aging and Cancer. \\ Tohoku University, Sendai, Japan; and 'Bioacoustics Research Laboratory, \\ University of Illinois, Urbana, IL, USA
}

(Received 6 November 1995: in final form 13 August 1996)

\begin{abstract}
The purpose of this study was to ultrasonically characterize infarcted human myocardial tissue at the microscopic level by scanning acoustic microscopy. Infarcted myocardial specimens from ten cases with acute myocardial infarction were studied. Specimens were formalin fixed, parafin embedded and sectioned to $10-\mu \mathrm{m}$ thickness. A specially developed scanning acoustic microscope system, operating in the 100- to 200-MHz ultrasound frequency range, was used for the measurements. The values of the attenuation constant were $0.94 \pm 0.04 \mathrm{~dB} / \mathrm{mm} / \mathrm{MHz}$ in normal myocardium, $0.71 \pm 0.12 \mathrm{~dB} / \mathrm{mm} / \mathrm{MHz}$ in degenerated myocardium, $0.88 \pm 0.47 \mathrm{~dB} / \mathrm{mm} / \mathrm{MHz}$ in granulation tissue and $1.75 \pm 0.11 \mathrm{~dB} / \mathrm{mm} / \mathrm{MHz}$ in fibresis. The values of sound speed were $1620.2 \pm 8.2 \mathrm{~m} / \mathrm{s}$ in normal myocardium, $1572.4 \pm 10.6 \mathrm{~m} / \mathrm{s}$ in degenerated myocardium, $1590.2 \pm 32.5 \mathrm{~m} / \mathrm{s}$ in granulation tissue and $1690.3 \pm 9.1 \mathrm{~m} / \mathrm{s}$ in fibrosis. The ultrasonic properties of the diseased myocardium at the microscopic level will provide important information for ultrasonic tissue characterization at the macroscopic level. Copyright (C) 1997 World Federation for Ultrasound in Medicine \& Biology.
\end{abstract}

Key Words: Ultrasound, Acute myocardial infarction, Myocardium, Ultrasonic tissue characterization, Acoustic microscope, Sound speed, Attenuation.

\section{INTRODUCTION}

Echocardiography has been widely used clinically for the detection of areas of infarcted myocardium and for evaluation of the degree of infarction, largely because of its noninvasiveness, reliability and repeatability. Wall-motion abnormalities, during and after myocardial infarction, can be detected by M-mode echocardiography or by centerline analysis of the two-dimensional echocardiogram (Hikichi and Tanaka 1981a; Moinihan et al. 1980; Parisi et al. 1980).

Ultrasonic tissue characterization of myocardium is also under development (Lattanzi et al. 1991, 1992; Miller et al. 1985; Milunski et al. 1989; Mimbs et al. 1977; Picano et al. 1990, 1993; Scorton et al. 1983). The need to evaluate local myocardial function has increased in proportion to the progress of interventional and surgical therapy for coronary artery disease

Address correspondence to: Yoshifumi Saijo, Department of Medical Engineering and Cardiology, Institute of Development. Aging and Cancer, Tohoku University, 4-1 Seiryo-machi, Aoba-ku, Sendai-shi, 980 Japan
(Ellis et al. 1995; Hannan et al. 1994). Tissue characterization studies have been reported, e.g., the detection of the cyclic variation of ultrasonic backscatter (Barzilai et al. 1990; Miller et al. 1985; Milunski et al. 1989; Mimbs et al. 1977; Vered et al. 1989), the analysis of the gray scale level of echography (Chandrasekaran et al. 1989; Parisi et al. 1982; Scorton et al. 1983); and the analysis of echo intensity by changing the echo sensitivity (Hikichi et al. 1981b; Tanaka et al. 1977, 1985). The results of these investigations suggest that the acoustic properties of infarcted myocardium can be different from the normal state. The mechanisms responsible for the changes at the microscopic level determined by using high-frequency ultrasound have not been fully discussed, although the acoustic properties of myocardium at the low $\mathrm{MHz}$ frequencies are affected by the inhomogeneities of the acoustic contrast at the microscopic level.

A specially developed scanning acoustic microscope (SAM) system (Okawai et al. 1987, 1988; Saijo et al. 1991) has been used to determine quantitatively the ultrasonic properties of the infarcted myocardium, 
because the structure of the infarcted area at the microscopic level can be observed using high-frequency ultrasound.

Measurements of the ultrasonic attenuation and sound speed were performed using the SAM system in the following tissue elements of infarcted myocardium: normal myocardium, degenerated myocardium, granulation tissue and fibrosis. Characteristics of the acoustic properties of infarcted myocardium are also discussed.

\section{METHODS}

Infarcted myocardial specimens were obtained from autopsy of ten cases with acute myocardial infarction. The specimens were formalin fixed, paraffin embedded and sectioned to approximately $10 \mu \mathrm{m}$ in thickness using a microtome. The tissue was sectioned in a plane parallel to the normal muscle fiber orientation. The specimens were mounted on glass slides but were not covered by coverslips while measurements were made with the SAM system. The paraffin was removed from the mounted sections by the graded alcohol method just before ultrasonic measurement.

Tissue elements of the infarcted myocardium were classified based upon the pathohistological findings into the following four types: normal myocardium, degenerated myocardium, granulation tissue and fibrosis (Fishbein et al. 1978). In the present study, the myocardium that seemed normal by optical microscopy, with the striation in the myocyte, was defined as normal myocardium. The myocyte in which the striation had disappeared and stained homogeneously by elastica-Masson staining was defined as degenerated myocardium. The tissue containing macrophage and histiocyte digesting the degenerated myocardium was granulation tissue. The bundle of collagen fiber stained green by elastica-Masson staining was defined as fibrosis. The infarcted area was diagnosed clinically by the electrocardiographic and echocardiographic findings, and the disease stage was determined from the onset of clinical symptoms, viz, chest pain, cold sweat and nausea. By pathological findings, the culprit region was apparent for all the patients involved in the present study, so the tissue sources were considered to be from acute to subacute stages of myocardial infarction.

Two-dimensional distributions of attenuation and sound speed can be obtained using the SAM system. Figure 1 is a block diagram of the SAM system. The ultrasonic transducers are illustrated in the figure. The

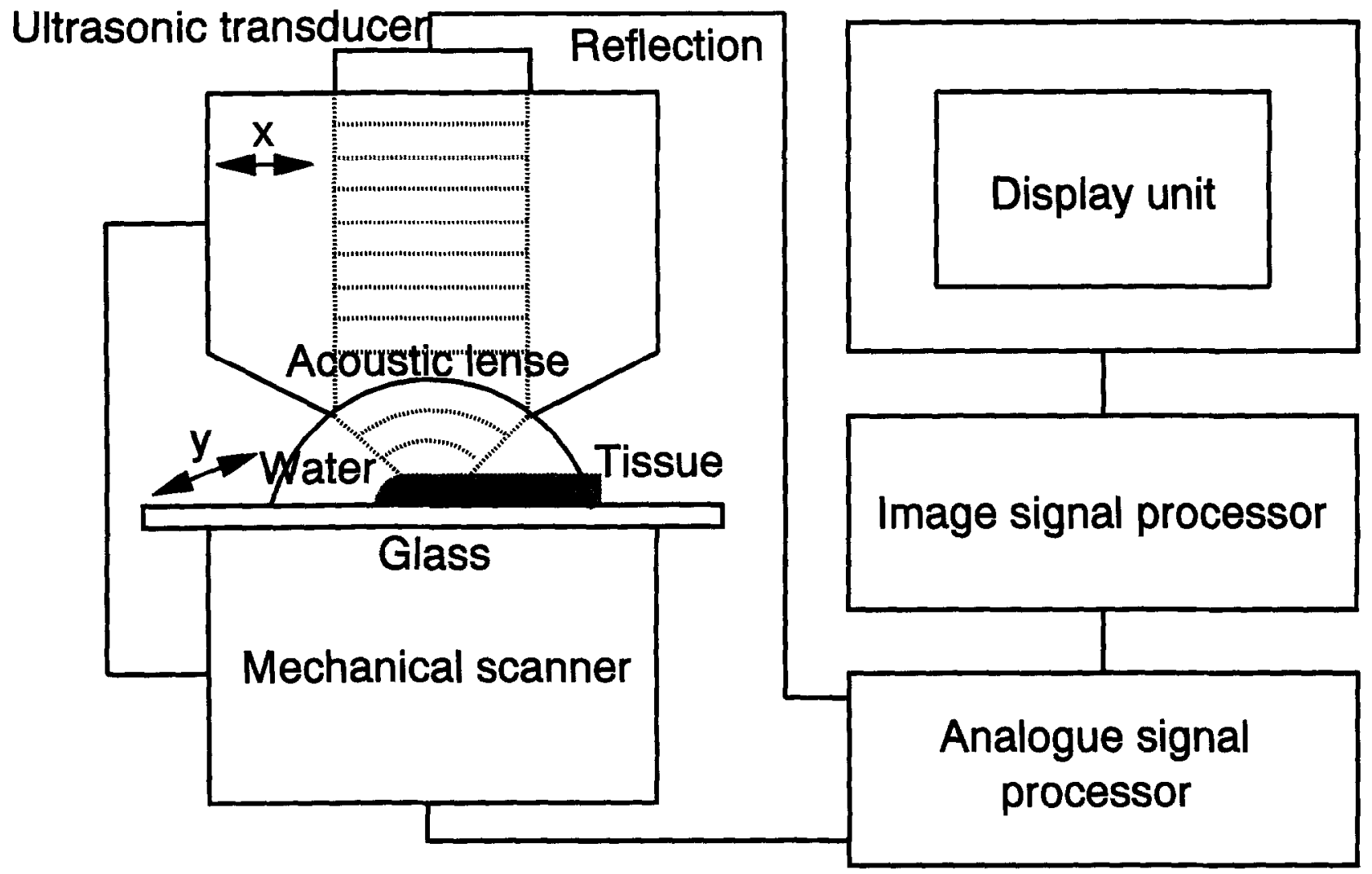

Fig. 1. Block diagram of the scanning acoustic microscope system. 
upper one is an acoustic focusing element consisting of a $\mathrm{ZnO}$ piezoelectric transducer with a sapphire lens. The aperture half angle is $30^{\circ}$ and focuses the acoustic beam with a $\frac{1}{2}$ power beam width lateral resolution from approximately $5 \mu \mathrm{m}$ at $200 \mathrm{MHz}$ to $10 \mu \mathrm{m}$ at $100 \mathrm{MHz}$. The lower one is a plane type transducer that is $6 \mathrm{~mm}$ in diameter wider than the scan width. In the present study, only the upper transducer was used for the reflection method. With the $10-\mu \mathrm{m}$ thick tissue specimen mounted on the glass slide, a threelayer structure of coupling medium, tissue section and glass slide is obtained. The thickness corresponds to $\frac{1}{2}-\frac{1}{3}$ wavelength, so the principle of interference can be used. Consequently, the reflection coefficient frequency characteristics exhibit a unique pattern, depending upon tissue thickness.

The mechanical scanner is arranged so that the ultrasonic beam is transmitted for every $4-\mu \mathrm{m}$ interval over a 2-mm scanned width. The number of sampling points is 480 in one scanning line, and $480 \times 480$ points make one frame within $4 \mathrm{~s}$.

The original signals for imaging produced in the analogue signal processor can be displayed on the CRT screen directly. However, the original signals are not sufficiently accurate values of attenuation or sound speed due to the lack of uniformity in section thickness. The image processor stores the original image signals in 16 frames: 11 frames of amplitude images in 10$\mathrm{MHz}$ steps in the range of $100-200 \mathrm{MHz}$, and five frames of phase images in 10- $\mathrm{MHz}$ steps in the range of $100-140 \mathrm{MHz}$. Figure 2 shows the frequency-amplitude and frequency-phase relationships, calculated when the specimen thickness is 8,9 and $10 \mu \mathrm{m}$. The thickness of the specimen is determined from the frequency-dependent characteristics of the amplitude and the phase of the received signals obtained at the same position for each of the 16 frames (Okawai et al. 1987, 1988). The slope of attenuation and the sound speed are calculated by the computer using eqns (1) and (2):

$$
\begin{gathered}
A=\frac{L}{f d} \\
c=\frac{1}{\frac{1}{c_{w}}-\frac{\phi}{2 \pi f d}}
\end{gathered}
$$
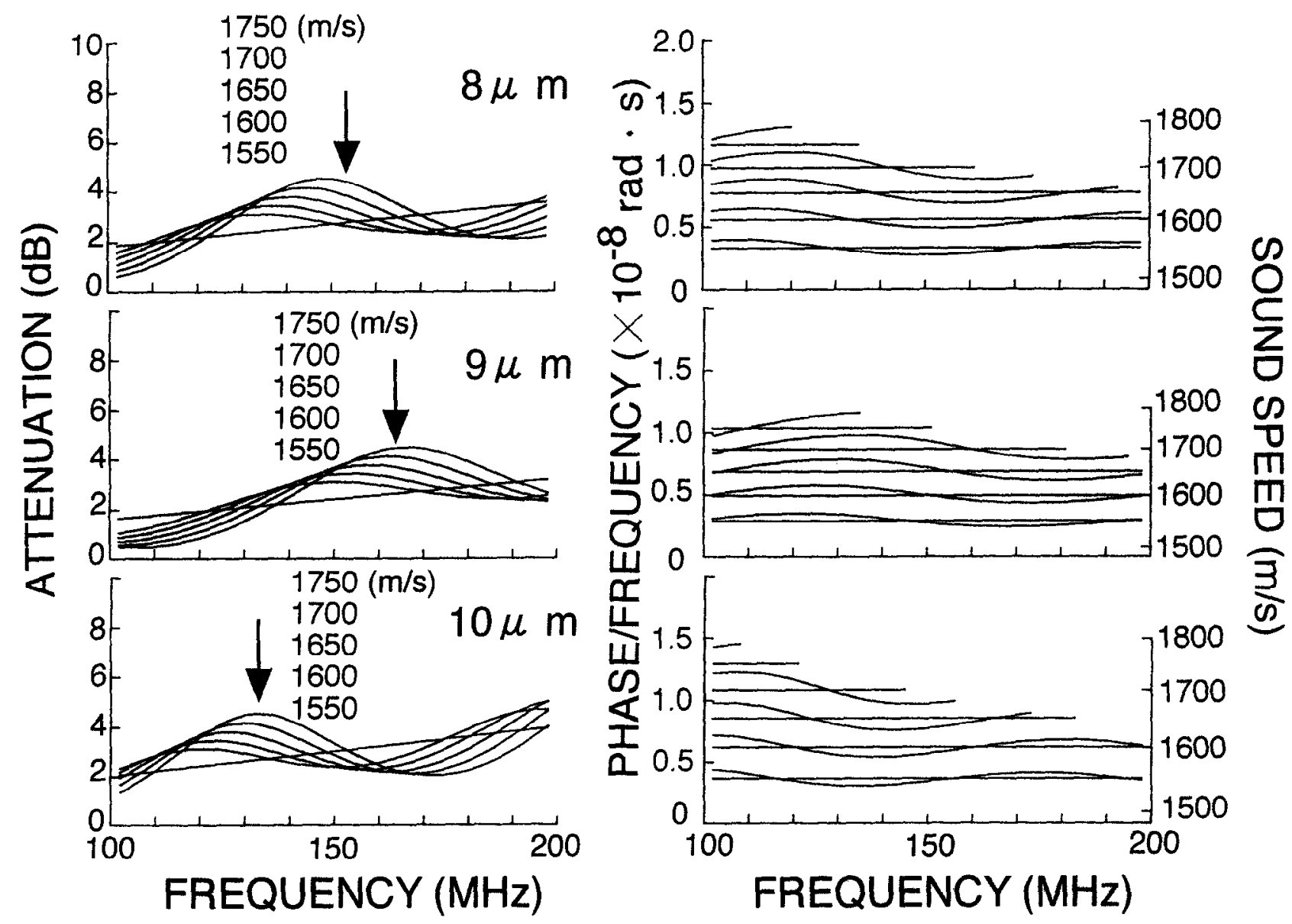

Fig. 2. Frequency-amplitude and frequency-phase relationships at sample thicknesses of 8,9 and $10 \mu \mathrm{m}$. 
Table 1. Relationship of color-coded scales and values of sound speed and attenuation constant.

\begin{tabular}{clr}
$\begin{array}{c}\text { Attenuation constant } \\
(\mathrm{dB} / \mathrm{mm} / \mathrm{MHz})\end{array}$ & Color & $\begin{array}{c}\text { Sound speed } \\
(\mathrm{m} / \mathrm{s})\end{array}$ \\
\hline $1.9 \sim$ & Red & $1690 \sim$ \\
$1.7 \sim 1.9$ & Magenta & $1670 \sim 1690$ \\
$1.5 \sim 1.7$ & Orange & $1650 \sim 1670$ \\
$1.3 \sim 1.5$ & Brown & $1630 \sim 1650$ \\
$1.1 \sim 1.3$ & Yellow & $1610 \sim 1630$ \\
$0.9 \sim 1.1$ & Green & $1590 \sim 1610$ \\
$0.7 \sim 0.9$ & Olive green & $1570 \sim 1590$ \\
$0.5 \sim 0.7$ & Cyan & $1550 \sim 1570$ \\
$0.3 \sim 0.5$ & Royal blue & $1530 \sim 1550$ \\
$0.1 \sim 0.3$ & Blue & $1510 \sim 1530$ \\
$\sim 0.1$ & Black & $\sim 1510$ \\
\hline
\end{tabular}

where $A$ is the slope of attenuation, $L$ is the amplitude of the reflected ultrasound, $f$ is the frequency used, $d$ is the thickness of the specimen, $c$ is the sound speed in the specimen, $c_{w}$ is the sound speed in the coupling medium and $\phi$ is the phase shift with respect to reflection from the glass surface. The step for the determination of thickness could involve 5\% error, so the attenuation values had 5\% error. The sound speed was determined as the relative value of the sound speed of water. The range of sound speed measurable by this method was between 1480 and $1690 \mathrm{~m} / \mathrm{s}$; therefore, the error of the sound speed would be $10 \mathrm{~m} / \mathrm{s}(5 \%$ of $210 \mathrm{~m} /$ s). The accuracy of the method was considered to be sufficient to discuss the quantitative values of the biological specimens.

A linear frequency dependence of the attenuation, in the ultrasonic frequency range of $100-200 \mathrm{MHz}$, was obtained from consideration of the interference of sound wave components (Okawai et al. 1987). The data of slope of attenuation and sound speed thus obtained were converted into color signals that corresponded to the values of the amplitude and phase, and were displayed on a color monitor as two-dimensional distribution patterns. Table 1 shows the relationship between color-coded scales and values of slope of attenuation and sound speed. Distilled water was used for the coupling medium, which was maintained between 20 and $22^{\circ} \mathrm{C}$ during the measurement procedure.

A neighboring section of the SAM specimen was stained with elastica-Masson's trichrome stain and used for optical microscopy. The region of interest (ROI) for acoustic microscopy was determined, as the single tissue element was included in the ROI, by the optical microscopic observations. The size of the ROI was $200 \times 200 \mu \mathrm{m}$ for optical microscopy and $50 \times$ 50 sampling points for acoustic microscopy. For each specimen, the ultrasonic attenuation constant and the sound speed in the four tissue elements were obtained from an average of the values in the ROI. The results for each tissue element, for all the specimens, are given as mean values $\pm 1 \mathrm{SD}$. The results for degenerated myocardium, granulation tissue and fibrosis were compared to the values in normal myocardium using analysis of variance by Fisher's protected least significant difference method.

The density of normal myocardium, degenerated myocardium and fibrosis was measured by the graded $\mathrm{CuSO}_{4}$ solution method to provide data on the specific acoustic impedance and elastic properties of the tissue elements. The sample was put in the $\mathrm{CuSO}_{4}$ solutions, prepared in different flasks as the concentrations were increased from $1.050 \mathrm{~g} / \mathrm{cm}^{3}, 1.051 \mathrm{~g} / \mathrm{cm}^{3}, 1.052 \mathrm{~g} /$ $\mathrm{cm}^{3} \ldots 1.100 \mathrm{~g} / \mathrm{cm}^{3}$. The density of the sample was determined when the sample did not float or sink in the solution.

\section{RESULTS}

Figure 3 shows the optical and acoustic images of specimen no. 8 of acute myocardial infarction. The green areas in the optical image and the red or magenta areas in the acoustic images represent fibrosis. The figure shows that the attenuation varies from 1.7-1.9 and over $1.9 \mathrm{~dB} / \mathrm{mm} / \mathrm{MHz}$ and the sound speed varies from $1670-1690$ and over $1690 \mathrm{~m} / \mathrm{s}$, respectively, in the fibrotic lesion. Both the attenuation and sound speed of the area of fibrosis are greater than those of normal myocardium, which is represented as yellow or green in the acoustic images.

Degenerated myocardium, which is observed in the adjacent area of the fibrotic lesion, is represented as cyan or royal blue in the acoustic images. These results indicate that the attenuation and sound speed in the area of degeneration are lower than those in normal myocardium.

Figures 4 and 5 are optical micrographs of the four kinds of tissue elements studied. Striation is observed in normal myocardium but does not appear in degenerated myocardium. Macrophages and histiocytes in the process of disposing of degenerated myocardium are observed in the granulation tissue. In the fibrotic lesion, the major component of the fibrosis is considered to be collagen since the fiber is stained green by elastica-Masson's stain. In Figs. 3 and 5, the collagen fibers are aligned along the long axis of the myocardial cell and the fibers are straight in the infarcted tissue in acute myocardial infarction.

Table 2 lists the average values of the slope of attenuation and sound speed measured in each specimen for the four types of tissue elements obtained from the cases with acute myocardial infarction. The blank entries imply the elements were not observed in the specimen. Table 2 also shows the mean and SD of 


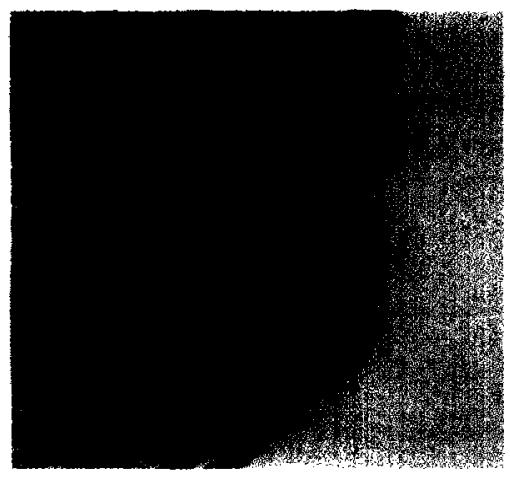

$\overleftrightarrow{500 \mu m}$ OPTICAL IMAGE

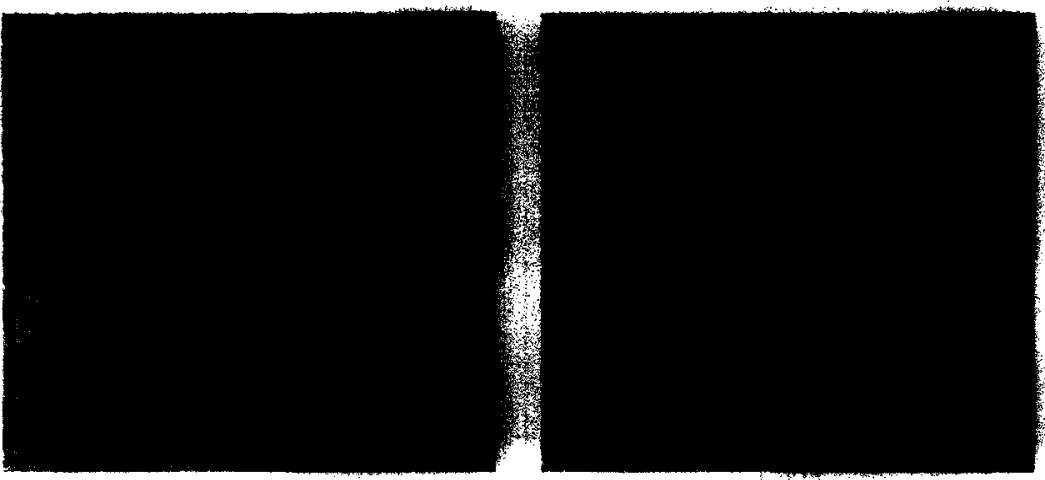

Sound Speed ACOUSTIC IMAGES

Fig. 3. Optical and acoustic images of a specimen of acute myocardial infarction.

the slope of attenuation and sound speed data for all specimens. The values in the degenerated myocardium are significantly lower than those in normal myocar$\operatorname{dium}(p<0.05)$. Both the attenuation and sound speed in fibrosis are significantly higher than those in normal myocardium $(p<0.01)$.

The SD of granulation tissue was so large that significant variance was not found in this study.

Table 3 shows the densities measured in three of the tissue elements. These were the same specimens as those from which sections were taken for examination under SAM. The density of granulation tissue could not be obtained, as a sufficient number of samples were not available.

\section{DISCUSSION}

In this study, the density of degenerated myocardium was less than that of normal myocardium. As shown in Fig. 4, the striation in the myocyte, which consists of actin and myosin filaments, has disappeared in the degenerated myocardium. The optical micrograph suggests that the linkage between actin and myosin is destroyed and that the intracellular structure be-

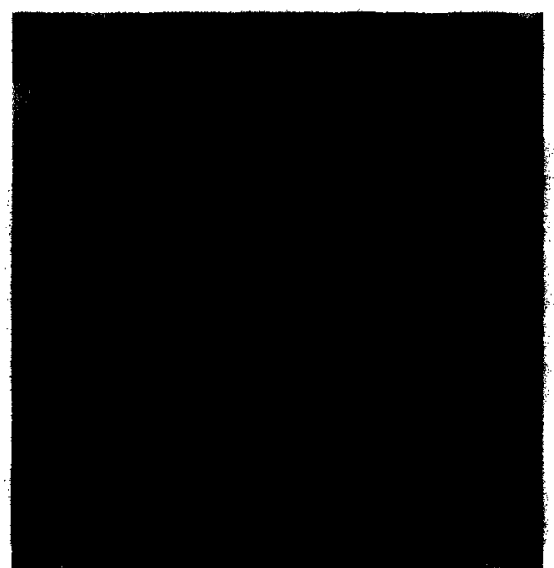

NORMAL

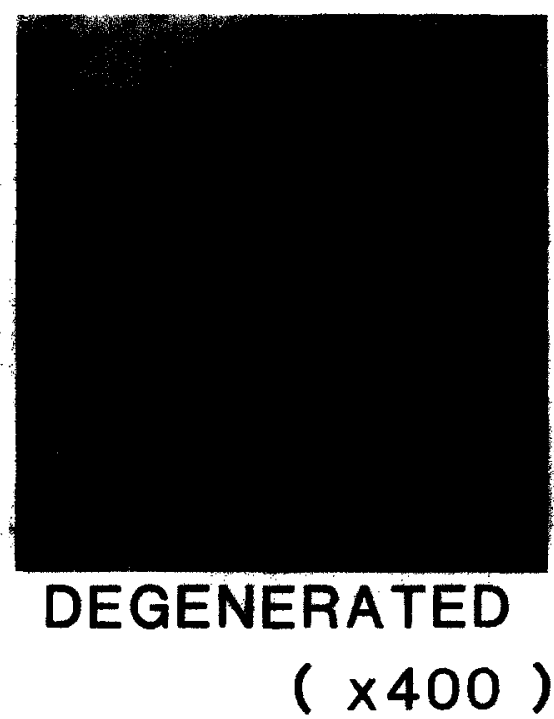

Fig. 4. Optical microscopic images of normal myocardium (left) and degenerated myocardium (right) $(\times 400$, elastica-Masson's trichrome stain). 


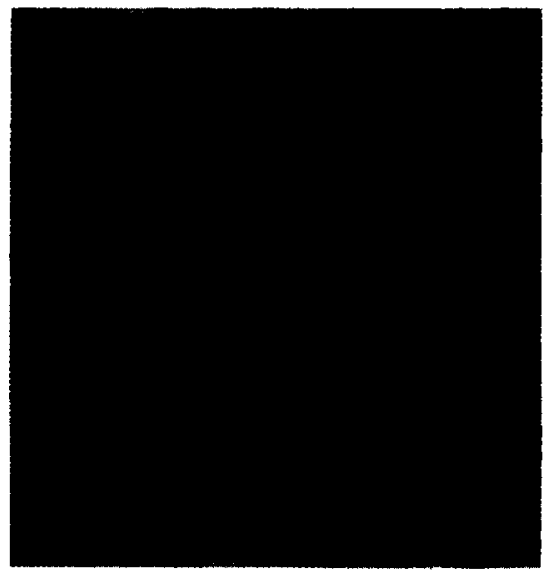

GRANULATION

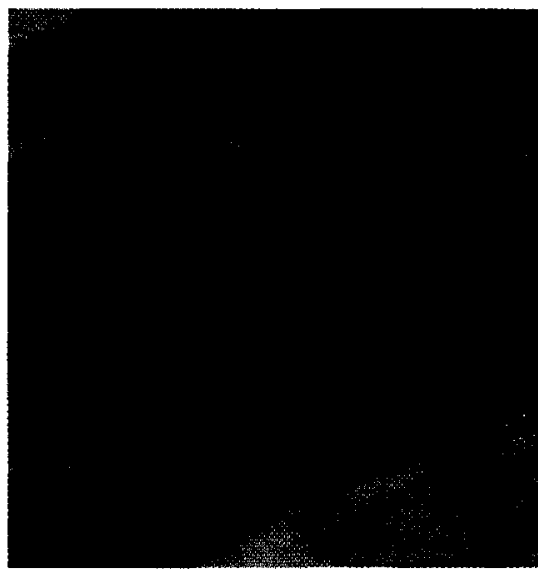

FIBROSIS

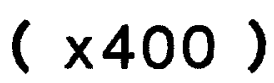

Fig. 5. Optical microscopic images of granulation tissue (left) and fibrosis (right) $(\times 400$, elastica-Masson's trichrome stain).

comes nearly homogeneous, as an acoustic medium, in the degenerated myocardium. Also, the size of each degenerated myocyte is larger than that of normal myocytes. Accordingly, the degenerated myocyte is understood to be swollen, and the density of the myocyte may become less than that of a normal one.

The area of fibrosis in acute myocardial infarction is organized mainly by collagen fibers. Consequently, the density of fibrosis is considered to be increased in proportion to the amount of collagen because of the high molecular weight of collagen ( 300000 or more).

The attenuation represents all losses, viz, scatter- ing and absorption processes. Scattering is considered to be related to structural features of the medium, exhibiting spatial distributions of characteristic acoustic impedance causing redirection of the wave energy out of the main beam. Absorption is intimately related to the macromolecular content of the medium whereby the acoustic energy is converted to heat. Thus, for structural, high-density, large molecular weight media, such as collagenous tissue, both scattering and absorption are substantial, leading to high attenuation. In degenerated myocardium, the cellular structure disappeared and the intracellular components appeared to

Table 2. Average values of the attenuation constant (ATT) and sound speed (SPD) in specimens of acute myocardial infarction.

\begin{tabular}{|c|c|c|c|c|c|c|c|c|}
\hline \multirow[b]{2}{*}{ No. } & \multicolumn{2}{|c|}{ Normal } & \multicolumn{2}{|c|}{ Degenerated } & \multicolumn{2}{|c|}{ Granulation } & \multicolumn{2}{|c|}{ Fibrosis } \\
\hline & ATT & SPD & ATT & SPD & ATT & SPD & ATT & SPD \\
\hline 1 & 0.95 & 1626 & 0.71 & 1572 & 0.83 & 1594 & 1.57 & 1676 \\
\hline 2 & 0.94 & 1625 & 0.72 & 1577 & 0.40 & 1553 & 1.85 & 1698 \\
\hline 3 & 0.94 & 1622 & 0.83 & 1580 & 0.99 & 1599 & 1.73 & 1685 \\
\hline 4 & 1.02 & 1632 & 0.57 & 1564 & 0.48 & 1558 & 1.86 & 1698 \\
\hline 5 & 1.00 & 1629 & 0.82 & 1574 & 1.71 & 1645 & 1.74 & 1690 \\
\hline 6 & 0.92 & 1620 & 0.52 & 1553 & & & 1.55 & 1677 \\
\hline 7 & 0.93 & 1615 & 0.84 & 1591 & & & 1.71 & 1682 \\
\hline 8 & 0.88 & 1611 & 0.62 & 1567 & & & 1.87 & 1702 \\
\hline 9 & 0.92 & 1614 & & & & & 1.76 & 1694 \\
\hline 10 & 0.87 & 1608 & & & & & 1.83 & 1696 \\
\hline Mean & 0.94 & 1620.2 & 0.71 & 1572.4 & 0.88 & 1590.2 & 1.75 & 1690.3 \\
\hline SD & 0.04 & 8.2 & 0.12 & 10.6 & 0.47 & 32.5 & 0.11 & 8.2 \\
\hline$p$ & - & - & 0.05 & 0.05 & NS & NS & 0.01 & 0.01 \\
\hline
\end{tabular}

$p$ compared to normal myocardium. 
Table 3. Values of sound speed, density, calculated specific acoustic impedance and bulk modulus of normal $(n=10)$, degenerated $(n=8)$ and fibrotic $(n=10)$ tissue elements (mean \pm SD).

\begin{tabular}{llccr}
\hline Tissue type & $\begin{array}{c}\text { Sound speed } \\
(\mathrm{m} / \mathrm{s})\end{array}$ & $\begin{array}{c}\text { Density } \\
\left(\mathrm{g} / \mathrm{cm}^{3}\right)\end{array}$ & $\begin{array}{c}\text { Specific acoustic } \\
\text { impedance }\left(\mathrm{Ns} / \mathrm{m}^{3}\right)\end{array}$ & $\begin{array}{c}\text { Bulk modulus } \\
\left(\mathrm{N} / \mathrm{m}^{2}\right)\end{array}$ \\
\hline Normal & $1620.2 \pm 8.2$ & $1.082 \pm 0.02$ & $1.75 \times 10^{6}$ & $2.84 \times 10^{9}$ \\
Degenerated & $1572.4 \pm 10.6$ & $1.074 \pm 0.04$ & $1.69 \times 10^{6}$ & $2.66 \times 10^{9}$ \\
Fibrosis & $1690.3 \pm 9.1$ & $1.092 \pm 0.02$ & $1.85 \times 10^{6}$ & $3.12 \times 10^{9}$ \\
\hline
\end{tabular}

become homogeneous and fluid-like. Accordingly, scattering is reduced due to the more uniform spatial distribution of the impedance, essentially loss of structure. The absorption is reduced, due to the low macromolecular content. Thus, attenuation is decreased.

The SD of the granulation tissue studied was very large, suggesting that it may not yield a unique value for the attenuation constant. Granulation tissue is comprised of various components of the tissue elements, viz, degenerated myocardium, which exhibits a low attenuation constant, and fibrocytes, fibroblasts, collagen fibers, etc, which have a considerably higher attenuation constant. For specimen no. 5 (Table 2), which contained a large number of cells, the mean value of the attenuation showed a broad range. Thus, attenuation may be considered to express the pathological feature of granulation tissue.

In this study, both attenuation and sound speed decreased in the initial stage, after which both properties increased with the time course of disease. Miller et al. (1985) reported similar effects from their work on dogs using low-frequency ultrasound. They reported that attenuation decreased initially following infarction as an edematous reaction, after which attenuation increased as scar formation. If the slope of the attenuation is linear in overall frequency, the data of Miller et al. (1985) and our results demonstrated the same phenomenon at different frequencies. However, the slope of attenuation may not be linear, because the structure of the biological tissue is so complicated that the scattering affects the frequency-attenuation relationship. Therefore, the data of Miller et al. (1985) and our results suggest that the macroscopic acoustic properties are affected by the microscopic properties obtained by acoustic microscopy.

Sound speed contains information on the physical and chemical states of the tissue components. Density $\rho$ and sound speed $c$ determine the characteristic acoustic impedance $Z$ of the tissue as:

$$
Z=\rho c
$$

The tissue used in this study was formalin fixed, and it is known that such a preparation increases the sound speed by $0.5 \%$ (Bamber et al. 1979). Alcohol dehydration is known to remove all fat from the tissue specimen. In the present study, a large amount of fat content was not observed in the ROI by optical microscopy, so the dehydration process may not have had a substantial influence on the specimen.

If the formalin fixation and alcohol dehydration had a negligible influence on the specimens, there are some different conditions between in vivo imaging behavior and the SAM measurement, viz, temperature, cardiac motion, cessation of blood flow, etc. Thus, the absolute values obtained in the present study may contain some errors. However, it is very meaningful for us to use the data when discussing the relative relationships of the acoustic properties among the different tissue elements. Therefore, the sound speed data measured in this study were applied to this equation when discussing the biological physical properties.

Table 3 shows the calculated acoustic impedance values.

On the assumption that the interface between two fluid-like media is infinite and plane, the $d B$ level of the relative reflected sound power is:

$$
\begin{aligned}
d B & =10 \log _{10} \frac{P_{r}}{P_{i}} \\
& =10 \log _{10} \frac{\left(Z_{a}-Z_{b}\right)^{2}}{\left(Z_{a}+Z_{b}\right)^{2}}
\end{aligned}
$$

where $P_{r}$ is the sound power reflected at the interface and $P_{i}$ is the incident sound power, both in medium $a, Z_{a}$ is the impedance of medium $a$ and $Z_{b}$ is the impedance of medium $b$.

The width of the ultrasound beam used in clinical echocardiography is about $1.5-2.0 \mathrm{~mm}$ in diameter in the focal area at a frequency of $3.5 \mathrm{MHz}$. As the size of fibrotic lesions is $>1 \mathrm{~cm}^{2}$ in acute myocardial infarction tissue, it is considered to be large enough to be modeled by simple reflection at the interface between normal myocardium and the region of infarction. Table 4 shows the simple $\mathrm{dB}$ level reflected sound power at the interface between pairs of these tissue elements. The level at the interface between degenerated myocar- 
dium and fibrosis is calculated as $-26.9 \mathrm{~dB}$ and is the greatest value among those considered in this study. The clinical echocardiography literature shows that strong echoes are observed at the area of scar in myocardial infarction (Parisi et al. 1982; Scorton et al. 1983). The dB level of reflected power at the interface between fibrosis and the other tissue elements may be proposed as one of the origin of the strong echo in the myocardial scar.

The interface of pairs of tissue elements is not completely smooth relative to a wavelength of the ultrasound used, and simple reflection may not always occur. Nevertheless, clinical echocardiography should be capable of detecting the fibrotic change of myocardial infarction. There may be another explanation, e.g., the acoustic impedance mismatch, or the inhomogeneity of the acoustic field.

The sound speed in a medium modeled as a fluid may be taken as:

$$
c=\sqrt{\frac{K}{\rho}}
$$

where $c$ is the sound speed, $K$ is the elastic bulk modulus and $\rho$ is the density.

As biological tissue is considered to be fluid-like, the sound speed of the tissue element is determined by its elasticity. Table 3 also shows the results of calculating bulk modulus from the velocity data. Here it is seen that the stiffness of fibrotic tissue increases $10 \%$ and that of degenerated myocardium decreases $6 \%$ in comparison with normal myocardium. Whittaker et al. (1991) have reported that the role of collagen fibers in acute myocardial infarction is to prevent expansion of the infarction. Mann et al. (1988) have pointed out that the frequency of left ventricular rupture is higher in the group that exhibits no remarkable increase of scar in myocardium. From the viewpoint of pathology, these two reports suggest that collagen fiber is a stiff tissue component and that the elasticity of collagenous fibrosis is large. Verdonk et al. (1992) and Hoffmeister et al. (1994) showed the anisotropy of ultrasonic velocity and elastic properties in myocardium. They dem-

Table 4. Simple dB levels of reflected power between pairs of tissue elements.

\begin{tabular}{lccc}
\hline & Normal & Degenerated & Fibrosis \\
\hline Normal & $*$ & -35.2 & -31.1 \\
Degenerated & -35.2 & $*$ & -26.9 \\
Fibrosis & -31.1 & -26.9 & $*$ \\
\hline
\end{tabular}

*, no calculated value. onstrated that velocity parallel to the myocardial fiber was faster than velocity perpendicular to the fiber.

Our study has shown that the sound speed of fibrosis perpendicular to the fiber is higher than in normal myocardium. Consequently, the elasticity along the fiber, greater than that of the other tissue elements, would protect the heart from overstretching. This physical property of the fibrosis thus protects the heart from cardiac rupture because of its greater stiffness.

\section{CONCLUSION}

Ultrasonic attenuation and sound speed were measured for four different types of tissue elements present in acute myocardial infarction. The attenuation constant and sound speed of degenerated myocardium were found to be very low compared with those of normal myocardium, and attenuation and sound speed were very high in fibrosis.

These results suggest that the ultrasonic properties of acute myocardial infarction were determined, to some extent, by density, intra- and intercellular structures and bulk modulus elasticity of the tissue elements. Acoustic microscopy provides information on physical properties, which cannot be obtained by optical microscopy, as well as on the pathological features of acute myocardial infarction.

The occurrence of strong echoes in the area of the fibrotic lesion in myocardial infarction may be supported by calculation of the level of simple reflected power between fibrosis and the other tissue elements in our study. It was also found that the myocardium becomes softer in degenerated tissues and stiffer in fibrotic tissues compared with normal myocardium.

\section{REFERENCES}

Bamber JC, Hill CR, King JA, Dunn F. Ultrasonic propagation through fixed and unfixed tissues. Ultrasound Med Biol 1979;5:159-165.

Barzilai B, Verad A, Mohr GA, et al. Myocardial ultrasonic backscatter for characterization of ischemia and reperfusion: Relationship to wall motion. Ultrasound Med Biol 1990;16:391-398.

Chandrasekaran K, Aylward PE, Fleagle SR, et al. Feasibility of identifying amyloid and hypertrophic cardiomyopathy with the use of computerized quantitative texture analysis of clinical echocardiographic data. J Am Coll Cardiol 1989;13:832-840.

Ellis SG, Cowley MJ, Whitlow PL, et al. Prospective case-control comparison of percutaneous transluminal coronary revascularization in patients with multivessel disease treated in 1986-1987 versus 1991: Improved in-hospital and 12-month results. J Am Coll Cardiol 1995;25:1137-1142.

Fishbein MC, Maclean D, Maroko PR. The histopathologic evaluation of myocardial infarction. Chest 1978;73:843-849.

Hannan EL, Kilburn H, Racz M, Shields S, Chassin MR. Improving the outcomes of coronary artery bypass surgery in New York State. JAMA 1994;271:761-766.

Hikichi H, Tanaka M. Detection of left ventricular asynergy in myocardial infarction by means of ultrasono-cardiotomography and M-mode echocardiography. Jpn Heart J 1981a;22:299-312.

Hikichi $H$, Tanaka $M$. Ultrasono-cardiotomographic evaluation of 
histological changes in myocardial infarction. Jpn Heart J $198 \mathrm{Ib} ; 22: 287-298$.

Hoffmeister BK, Verdonk ED, Wickline SA, Miller JG. Effect of collagen on the anisotropy of quasi-longitudinal mode ultrasonic velocity in fibrous soft tissues: A comparison of fixed tendon and fixed myocardium. J Acoust Soc Am 1994;96:1957-1964.

Lattanzi F, Spirito P, Picano E, et al. Qualitative assessment of ultrasonic myocardial reflectivity in hypertrophic cardiomyopathy. J Am Coll Cardiol 1991; 17:1085-1090.

Lattanzi F, Bello VD, Picano E, et al. Normal ultrasonic myocardial reflectivity in athletes with increased left ventricular mass. Circulation 1992;85:1828-1834.

Mann JM, Roberts WC. Rupture of the left ventricular free wall during acute myocardial infarction: Analysis of 138 necropsy palients and comparison with 50 necropsy patients with acute myocardial infarction without rupture. Am J Cardiol 1988; 62:847-859.

Miller JG, Perez JE, Sobel BE. Ultrasonic characterization of myocardium. Prog Cardiovasc Dis 1985;28:85-110.

Milunski MR, Mohr GA, Perez JE, et al. Ultrasonic tissue characterization with integrated backscatter: Acute myocardial ischemia, reperfusion, and stunned myocardium in patients. Circulation 1989;80:491-503.

Mimbs JW, Yuhas DE, Bauwens D, Miller JG, Sobel BE. Detection of myocardial infarction in vitro based on altered attenuation of ultrasound. Circ Res 1977;41:192-198.

Moinihan PF, Parisi AF, Feldman CL. Quantitative detection of regional left ventricular contraction abnormalities by two-dimensional echocardiography: I. Analysis of methods. Circulation $1980 ; 63: 752-760$

Okawai H, Tanaka M, Chubachi N, Kushibiki J. Non-contact simultaneous measurement of thickness and acoustic properties of a biological tissue using focused wave in a scanning acoustic microscope. Jpn J Appl Physiol 1987;26:52-54.

Okawai H, Tanaka M. Dunn F, Chubachi N, Honda K. Qualitative display of acoustic properties of the biological tissue elements. Acoustical Imaging 1988; 17:193-201.

Parisi AF, Moinihan PF, Folland ED, Feldman CL. Quantitative detection of regional left ventricular contraction abnormalities by two-dimensional echocardiography: II. Accuracy in coronary artery disease. Circulation 1980;63:761-767.

Parisi AF, Nieminen M. O'Boyle JE, et al. Enhanced detection of the evolution of tissue changes after acute myocardial infarction using color-encoded two-dimensional echocardiography. Circulation $1982 ; 66: 764770$.

Picano E, Pelosi G, Marzilli $\mathrm{M}$, et al. In vivo quantitative ultrasonic evaluation of myocardial fibrosis in man. Circulation 1990; 81:58-64.

Picano E, Faletra F, Marini C, et al. Increased echodensity of transiently asynergic myocardium in humans: A novel echocardiographic sign of myocardial ischemia. J Am Coll Cardiol 1993;21:199-207.

Saijo Y, Tanaka M, Okawai H, Dunn F. The ultrasonic properties of gastric cancer tissues obtained with a scanning acoustic microscope system. Ultrasound Med Biol 1991;17:709-714.

Scorton DJ, Melton HE, Pandian NG, et al. Detection of acute myocardial infarction in closed-chest dogs by analysis of regional two-dimensional echocardiographic gray-level distributions. Circ Res 1983;52:36-44.

Tanaka M, Terasawa $Y$, Hikichi $H$. Qualitative evaluation of the heart tissue by ultrasound. J Cardiogr 1977;7:515-530.

Tanaka M, Nitta S, Nitta K, et al. Non-invasive estimation by cross sectional echocardiography of myocardial damage in cardiomyopathy. Br Heart J 1985;53:137-152.

Verdonk ED, Wickline SA, Miller JG. Anisotropy of ultrasonic velocity and elastic properties in normal human myocardium. $\mathrm{J}$ Acoust Soc Am 1992;92:3039-3050.

Vered Z, Mohr GA, Barzilai B, et al. Ultrasound integrated tissue characterization of remote myocardial infarction in human subjects. J Am Coll Cardiol 1989;13:84-91.

Whittaker P, Boughner DR, Kloner RA. Role of collagen in acute myocardial infarct expansion. Circulation 1991;84:2123-2134. 